\title{
A transfer principle for second-order arithmetic, and applications
}

\author{
MERLIN CARL \\ ASGAR JAMNESHAN
}

\begin{abstract}
In the theory of conditional sets, many classical theorems from areas such as functional analysis, probability theory or measure theory are lifted to a conditional framework, often to be applied in areas such as mathematical economics or optimization. The frequent experience that such theorems can be proved by 'conditionalizations' of the classical proofs suggests that a general transfer principle is in the background, and that formulating and proving such a transfer principle would yield a wealth of useful further conditional versions of classical results, in addition to providing a uniform approach to the results already known. In this paper, we formulate and prove such a transfer principle based on second-order arithmetic, which, by the results of reverse mathematics, suffices for the bulk of classical mathematics, including real analysis, measure theory and countable algebra, and excluding only more remote realms like category theory, set-theoretical topology or uncountable set theory, see eg the introduction of Simpson [47]. This transfer principle is then employed to give short and easy proofs of conditional versions of central results in various areas of mathematics, including theorems which have not been proven by hand previously such as Peano existence theorem, Urysohn's lemma and the Markov-Kakutani fixed point theorem. Moreover, we compare the interpretation of certain structures in a conditional model with their meaning in a standard model.
\end{abstract}

2010 Mathematics Subject Classification 03C90,03F35 (primary); 28B20,54C65 (secondary)

Keywords: second-order arithmetic, conditional set theory, transfer principle

\section{Introduction}

Fixing a probability space $(\Omega, \mathcal{F}, \mathbb{P})$, one can distinguish between probabilistic and deterministic objects such as a deterministic real number which is an element of $\mathbb{R}$ and a random real number which is a measurable function $x: \Omega \rightarrow \mathbb{R}$. Extending such reasoning, one might speak of a 'random', 'stochastic', 'measurable' or 'conditional' 
version of a 'deterministic', 'classical' or 'standard' theorem which expresses a randomization of its statement. To illustrate, a conditional version of the BolzanoWeierstraß theorem states that for every sequence $\left(x_{k}\right)$ of random real numbers such that $x=\lim \sup x_{k}<\infty$ almost surely, there exists a strictly increasing sequence $n_{1}<n_{2}<\ldots$ of integer-valued random variables such that $x_{n_{k}}$ converges almost surely to $x$. Experience has shown that many classical theorems have such conditional analogues such as the Heine-Borel theorem, the Hahn-Banach extension and separation theorems and the Brouwer fixed point theorem, see eg Cheridito et al [10], Drapeau et al [15, 18], Filipovic et al [19], and Jamneshan et al [36, 38] for an account. It is thus tempting to aim for a general transfer principle that allows one to 'import' classical theorems into a conditional setting.

In Section 3, we prove such a transfer principle based on second-order logic. Strong arguments have been put forward in favor of the claim that second-order logic is a satisfying formal framework for the bulk of classical mathematics, see eg the introduction to Simpson [47]; this was impressively confirmed by the results of reverse mathematics. More precisely, we prove that any consequence of the second-order axiomatic system of arithmetical comprehension $\mathrm{ACA}_{0}$, which has a second-order comprehension axiom for formulas in which all quantifiers range over natural numbers (see Simpson [47]), also holds conditionally. To this end, we show (i) that the axioms of $\mathrm{ACA}_{0}$ hold in the structure with first order part $L^{0}(\mathbb{N})$ and second-order part its conditional power set $\mathcal{P}$ with truth value $\Omega$, and (ii) that truth value $\Omega$ is preserved by the usual deduction rules of second-order predicate calculus. To this end, a conditional element relation between $L^{0}(\mathbb{N})$ and $\mathcal{P}$ is introduced. We expect that, with a certain amount of extra technical effort, this can be extended to a considerably stronger transfer principle for full second-order logic.

In Section 4, we then discuss consequences of the transfer principle. We verify that the transfer principle yields a conditional version of a whole class of classical theorems many of which were previously proved by hand ${ }^{1}$. As new consequences, we obtain conditional versions of the Peano existence theorem, Urysohn's lemma, the existence of an orthonormal basis and the Markov-Kakutani theorem.

A conditional version of a classical theorem is oftentimes also a theorem about a more involved situation in a classical setting. For instance, the above conditional version of the Bolzano-Weierstraß-theorem is also a classical theorem securing existence of measurably parametrized almost surely converging subsequences of an almost surely

\footnotetext{
${ }^{1}$ The latter practice provides some useful insight into the transfer process.
} 
upper bounded sequence of real-valued measurable functions ${ }^{2}$. We will systematically investigate a standard interpretation of a conditional version of a classical structure or theorem in Section 4. As a result, bridges to the analysis in $L^{0}(\mathbb{R})$-modules (eg Cheredito et al [10], Filipovic et al [19]) and set-valued analysis (eg Rockafellar and Wets [45]) are built.

We will be also interested in the reverse direction. Namely, it can be verified that certain classical structures have a useful interpretation in a conditional model. For example, the standard space $L^{0}(\mathbb{R})$ of real-valued Borel measurable functions on a $\sigma$-finite measure space $(\Omega, \mathcal{F}, \mu)$ modulo almost everywhere equality are the real numbers in a conditional model. More generally, if $E$ is a complete separable metric space and $L^{0}(E)$ is the space of $E$-valued Borel measurable functions on $\Omega$ modulo almost everywhere equality, we show that $L^{0}(E)$ can be identified with a complete separable metric space in a conditional model of $\mathrm{ACA}_{0}$. The space $L^{0}(E)$ reflects a measurable parametrization of the elements of $E$ relative to a base space $(\Omega, \mathcal{F}, \mu)$. Such a measurable parametrization is a constituent part of a conditional model ${ }^{3}$. From an external perspective, one may view the transfer principle as a device which parametrizes classical theorems in a measurable way relative to a fixed measure space. In particular, the application of a conditional version of classical theorems preserves measurability, and thus provides an alternative to uniformization theorems (measurable selection lemmas) in descriptive set theory (see eg Kechris [40], Molchanov [44], or Rockafellar and Wets [45]), whenever one restricts attention to almost everywhere Borel selections. For example, we show that a maximum theorem in a conditional model is equivalent to a maximum theorem for normal integrands. In particular, compact subsets of a Euclidean space in a conditional model are uniquely related to compact-valued maps.

There is a practical interest in such model-theoretic results since the transfer principle is a highly efficient tool which replaces the tedious work of proving by hand conditional versions of classical results which are relevant in applications. Existing areas of application include probability (Jamneshan et al [36]), mathematical economics (Backhoff and Horst [3], Bielecki et al [5], Cheridito et al [8], Drapeau and Jamneshan [14], Filipovic et al [20], Frittelli and Maggis [23, 24], Hansen and Richard [31], and Kabanov and Stricker [39]), stochastic optimization (Cheridito and $\mathrm{Hu}$ [9], Cheridito and Stadje [11], and Jamneshan et al [37]), set-valued analysis and measurable selection theory

\footnotetext{
${ }^{2}$ In this classical form under the name of a 'randomized', 'stochastic' or 'measurable' version of the Bolzano-Weierstraß-theorem this statement is proved in Föllmer and Schied [21] and Kabanov and Stricker [39], motivated by applications in mathematical economics.

${ }^{3}$ Therefore conditional models might equally be called 'stochastic' or 'measurable' models of an axiomatic system.
} 
(Jamneshan et al [37] and Jamneshan and Zapata [38]), Lebesgue-Bochner spaces and vector duality (Drapeau et al [17] and Grad and Jamneshan [26]), and probabilistic analysis (Guo [27, 28], Guo et al [29], Guo and Zhang [30], and Jamneshan and Zapata [38]).

Conditional set theory (Drapeau et al [15]), which is used to build a 'conditional' model of $\mathrm{ACA}_{0}$, is conceptually closely related to Boolean-valued models and topoi of sheaves, see Jamneshan [35]. In fact, a conditional model of $\mathrm{ACA}_{0}$ is a Boolean-valued model of $\mathrm{ACA}_{0}$ by changing to the measure algebra associated to an underlying base space $(\Omega, \mathcal{F}, \mu)$. The constructive approach of second-order arithmetic permits us to explore the semantics in a Boolean-valued model, and through this understanding build a relationship to structures and theorems in a standard model, and thus facilitate applications.

In Aviles and Zapata [2], a categorical equivalence between conditional sets and a certain class of Boolean-valued sets is discussed. The correspondence is based on ZFC set theory rather than second-order arithmetic. We believe that our transfer principle is of independent interest, as (1) it is an explicitly formulated theorem; (2) second-order arithmetic allows a more direct and convenient modeling of relevant mathematical notions than set theory; for example, natural numbers and real numbers are treated as primitive objects and not as complicated sets; (3) it is not argued in [2] how one can deduce a transfer principle from a categorical equivalence and (4) the correspondence in [2, Theorem 3.1] excludes local subsets which are necessary to prove a transfer principle as shown in the proof of Theorem 3.4 below.

The remainder of this paper is organized as follows. In Section 2, we set up the stage and collect relevant notions from conditional set theory. In Section 3, we prove a transfer principle for $\mathrm{ACA}_{0}$. In Section 4, we discuss consequences and the applicability of the transfer principle. We conclude by a discussion of potential extensions of the transfer principle in Section 5.

Acknowledgement. Asgar Jamneshan gratefully acknowledges financial support from DFG project KU 2740/2-1.

\section{Preliminaries}

In the language $L_{2}$ of second-order arithmetic (see Simpson [47] for an introduction), we distinguish between number variables, traditionally written in lower-case Latin letters $x, y, z, \ldots$ and set variables, usually written as upper-case Latin letters $X, Y, Z, \ldots$. 
Moreover, we have two constant symbols 0 and 1, two binary function symbols + and - and a binary relation symbol $<$. Between numbers and sets exists an element relation $\epsilon$. The first-order terms are number variables, constant symbols and expressions of the form $t_{1}+t_{2}$ and $t_{1} \cdot t_{2}$ for first-oder terms $t_{1}$ and $t_{2}$. The atomic formulas are $t_{1}=t_{2}, t_{1}<t_{2}$ and $t_{1} \in X$ where $t_{1}$ and $t_{2}$ are first-order terms and $X$ is a set variable. The remaining formulas are obtained from atomic formulas by the use of propositional connectives and number and set quantifiers.

The axiomatic system of arithmetical comprehension $\left(\mathrm{ACA}_{0}\right)$ consists of the axioms for discretely ordered semirings, together with the second-order induction principle and an axiomatic scheme saying that for any second-order formula $\phi$ containing only first-order quantifiers there is a set of all natural numbers of which $\phi$ holds, see [47]. By the cumulative results of reverse mathematics, $\mathrm{ACA}_{0}$ is a sufficient axiomatic basis for a great number of theorems from classical mathematics; many examples can be found in [47].

Throughout fix a $\sigma$-finite measure space $(\Omega, \mathcal{F}, \mu)$, and let $\mathcal{N}$ denote its $\sigma$-ideal of null sets. We always identify $A, B \in \mathcal{F}$ whenever $A \Delta B \in \mathcal{N}$ where $\Delta$ denotes symmetric difference. The resulting quotient Boolean algebra has the following relevant properties:

- Completeness: Any family in $\mathcal{F}$ has a union and an intersection in $\mathcal{F}$.

- Countable chain condition: Any pairwise disjoint family in $\mathcal{F}$ is at most countable.

See eg Givant and Halmos [25, Chapter 31] for a reference. We will always identify two functions $x$ and $y$ on $\Omega$ with the same codomain if $\{\omega: x(\omega) \neq y(\omega)\} \in \mathcal{N}$. For a function $x$ on $\Omega$ and $A \in \mathcal{F}$, we write $x \mid A$ for the restriction of $x$ to $A$. For a Polish space $E$, let $L^{0}(E)$ denote the space of Borel functions $x: \Omega \rightarrow E$. In particular, $L^{0}(\mathbb{R})$ denotes the space of real-valued measurable functions, and $L^{0}(\mathbb{N}), L^{0}(\mathbb{Z})$ and $L^{0}(\mathbb{Q})$ denote its subsets of functions with values in $\mathbb{N}=\{0,1, \ldots\}$, the integers $\mathbb{Z}$ and the rational numbers $\mathbb{Q}$ respectively. Recall that $L^{0}(\mathbb{R})$ is a Dedekind complete Riesz lattice where addition, multiplication and order are defined pointwise, see eg Fremlin [22] for a reference. All inequalities between real-valued measurable functions shall be understood in the almost everywhere sense. By an abuse of language, we also denote by 0 and 1 the functions with constant values 0 and 1 respectively. For a measurable partition $\left(A_{k}\right)$ and a countable family $\left(x_{k}\right)$ in $L^{0}(E)$ for some Polish space $E$, we write $\sum_{k} x_{k} \mid A_{k}$ for the unique element $x \in L^{0}(E)$ such that $x\left|A_{k}=x_{k}\right| A_{k}$ for all $k$.

We introduce the conditional power set of $L^{0}(\mathbb{N})$, see Drapeau et al [15] for an introduction to conditional set theory. 
Definition 2.1 A set $N \subset L^{0}(\mathbb{N})$ is said to be stable under countable concatenations, or stable for short, if it is not empty and $\sum_{k} n_{k} \mid A_{k} \in N$ for all measurable partitions $\left(A_{k}\right)$ and every countable family $\left(n_{k}\right)$ in $N$. The conditional power set of $L^{0}(\mathbb{N})$ is the collection

$$
\mathcal{P}:=\left\{N \mid A: N \subset L^{0}(\mathbb{N}) \text { is stable, and } A \in \mathcal{F}\right\}
$$

where $N \mid A:=\{n \mid A: n \in N\}$. We write $N \mid \emptyset=\{*\}$.

We define a concatenation of a countable family $\left(N_{k} \mid B_{k}\right)$ in $\mathcal{P}$ and a measurable partition $\left(A_{k}\right)$ by

$$
\sum_{k}\left(N_{k} \mid B_{k}\right)\left|A_{k}:=\left(\sum_{k} N_{k} \mid A_{k}\right)\right| \cup_{k}\left(A_{k} \cap B_{k}\right)
$$

where

$$
\sum_{k} N_{k} \mid A_{k}:=\left\{\sum_{k} n_{k} \mid A_{k}: n_{k} \in N_{k} \text { for each } k\right\}
$$

Let us define conditional intersection and conditional complement. We follow the presentation in Jamneshan et al [36, Section 2]. Let $N|A, M| B \in \mathcal{P}$. The conditional intersection of $N \mid A$ and $M \mid B$ is defined as

$$
N|A \sqcap M| B:=V \mid C
$$

where:

$$
\begin{aligned}
& C:=\cup\left\{C^{\prime} \in \mathcal{F}: C^{\prime} \subset A \cap B, N\left|C^{\prime} \cap M\right| C^{\prime} \neq \emptyset\right\} \\
& V:=\left\{n \in L^{0}(\mathbb{N}): n|C \in N| C \cap M \mid C\right\}
\end{aligned}
$$

The conditional complement of $N \mid A$ is the conditional subset

$$
(N \mid A)^{\sqsubset}:=W \mid D
$$

where:

$$
\begin{aligned}
& D:=\cup\left\{D^{\prime} \in \mathcal{F}: \exists n \in L^{0}(\mathbb{N}) \text { such that } n|E \notin N| E\right. \\
& \left.\quad \text { for all } E \subset D^{\prime} \cap A \text { with } \mu(E)>0\right\} \\
& W:=\left\{n \in L^{0}(\mathbb{N}): n|E \notin N| E \text { for all } E \subset D \cap A \text { with } \mu(E)>0\right\}
\end{aligned}
$$

By applying an exhaustion argument, it can be derived from stability that $C$ and $D$ are attained, and it can be checked that $V$ and $W$ are stable sets as well. We conclude that the conditional intersection and conditional complement are well defined, see eg [36] for a complete argument. 
We introduce a conditional element relation between $L^{0}(\mathbb{N})$ and its conditional power set $\mathcal{P}$ which is a new ingredient in conditional set theory.

Definition 2.2 The conditional element relation is the function:

$$
\begin{aligned}
i & : L^{0}(\mathbb{N}) \times \mathcal{P} \rightarrow \mathcal{F} \\
i(n, N \mid A) & :=\cup\left\{A^{\prime} \in \mathcal{F}: A^{\prime} \subset A, n\left|A^{\prime} \in N\right| A^{\prime}\right\}
\end{aligned}
$$

The union of $\mathcal{G}:=\left\{A^{\prime} \in \mathcal{F}: A^{\prime} \subset A, n\left|A^{\prime} \in N\right| A^{\prime}\right\}$ is attained. Indeed, from Givant and Halmos [25, Section 30, Lemma 1] we know that there exists a countable family $\left(B_{k}\right)$ in $\mathcal{G}$ such that $A_{*}=\cup_{k} B_{k}=\cup \mathcal{G}$. Let $A_{1}=B_{1}$ and $A_{k}=B_{k} \cap\left(A_{1} \cup A_{2} \cup \ldots \cup A_{k-1}\right)^{c}$ for $k \geqslant 2$ which defines a measurable partition of $A_{*}$. Since $A_{k} \in \mathcal{G}$ for all $k$, the claim follows from stability of $N$. Further, it can be directly checked that:

$$
i\left(\sum_{k} n_{k}\left|A_{k}, N\right| A\right)=\cup_{k}\left(i\left(n_{k}, N \mid A\right) \cap A_{k}\right)
$$

\section{A Transfer Principle for $\mathrm{ACA}_{0}$}

Our aim is to prove that every consequence of $\mathrm{ACA}_{0}$ holds in the structure $\mathcal{S}:=$ $\left(L^{0}(\mathbb{N}), \mathcal{P},+, \cdot, 0,1,<, i\right)$ with truth value $\Omega$, ie that $\mathcal{S}$ is a 'conditional model' of $\mathrm{ACA}_{0}$. We start by explaining the evaluation of terms and formulas in $\mathcal{S}$.

Let $V_{1}$ denote the collection of all number variables and let $V_{2}$ denote the collection of all set variables. Let $\beta$ be a function with domain $V_{1} \cup V_{2}$ such that $\beta: V^{1} \rightarrow L^{0}(\mathbb{N})$ and $\beta: V^{2} \rightarrow \mathcal{P}$, and let $t$ be a first-order term. Such a $\beta$ is called a 'conditional assignment'. Then $[t]^{\beta}$, the $\beta$-evaluation of $t$, is defined recursively as:

- $[a]^{\beta}=a$ for $a \in\{0,1\}$

- $[x]^{\beta}=\beta(x)$ for $x \in V_{1}$

- $[X]^{\beta}=\beta(X)$ for $X \in V_{2}$

- $\left[t_{0} \circ t_{1}\right]^{\beta}=\left[t_{0}\right]^{\beta} \circ\left[t_{1}\right]^{\beta}$ for $\circ \in\{+, \cdot\}$

For first-order terms $t_{0}$ and $t_{1}$ and a second-order variable $X$, the conditional $\beta$ evaluation of atomic formulas is defined by:

- $\left[t_{0}=t_{1}\right]^{\beta}=\left\{\omega:\left[t_{0}\right]^{\beta}(\omega)=\left[t_{1}\right]^{\beta}(\omega)\right\}$

- $\left[t_{0}<t_{1}\right]^{\beta}=\left\{\omega:\left[t_{0}\right]^{\beta}(\omega)<\left[t_{1}\right]^{\beta}(\omega)\right\}$

- $\left[t_{1} \in X\right]^{\beta}=i\left(\left[t_{1}\right]^{\beta}, \beta(X)\right)$ 
The conditional $\beta$-evaluation of composite and quantified formulas is defined by:

- $[\phi \wedge \psi]^{\beta}=[\phi]^{\beta} \cap[\psi]^{\beta}$

- $[\neg \phi]^{\beta}=\left([\phi]^{\beta}\right)^{c}$

- $[\exists x \phi(x)]^{\beta}=\cup_{n \in L^{0}(\mathbb{N})}[\phi(x)]^{\beta\left[\frac{n}{x}\right]}$

- $[\exists X \phi(X)]^{\beta}=\cup_{N \mid A \in \mathcal{P}}[\phi(X)]^{\beta\left[\frac{N \mid A}{X}\right]}$

The remaining composite and quantification formulas are defined in the obvious way.

We have the following maximum principle, also known from Boolean-valued models, see eg Bell [4, Chapter 1].

Proposition 3.1 Let $\beta$ be a conditional assignment and let $\phi$ and $\psi$ be formulas in $L_{2}$. Then there exist $n \in L^{0}(\mathbb{N})$ and $N \mid A \in \mathcal{P}$ such that:

$$
\begin{gathered}
{[\exists x \phi(x)]^{\beta}=[\phi(x)]^{\beta\left[\frac{n}{x}\right]}} \\
{[\exists X \psi(X)]^{\beta}=[\psi(X)]^{\beta\left[\frac{N \mid A}{X}\right]}}
\end{gathered}
$$

Proof We may assume that $[\exists x \phi(x)]^{\beta}=\Omega$. We find a countable family $\left(A_{k}\right)=$ $\left([\phi(x)]^{\beta\left[\frac{n_{k}}{x}\right]}\right)$ such that $\cup_{k} A_{k}=[\exists x \phi(x)]^{\beta}$. Form a measurable partition from $\left(A_{k}\right)$, still denoted by $\left(A_{k}\right)$. Put $n=\sum_{k} n_{k} \mid A_{k}$. Then it holds that

$$
[\phi(x)]^{\beta\left[\frac{n}{x}\right]} \supset[\phi(x)]^{\beta\left[\frac{n_{k}}{x}\right]} \cap\left[n_{k}=n\right]^{\beta}
$$

for all $k$ which implies $[\phi(x)]^{\beta\left[\frac{n}{x}\right]}=[\exists x \phi(x)]^{\beta}$. The second claim can be shown analogously by using concatenations in $\mathcal{P}$.

We will now adapt the usual notion of the correctness of a sequent to the conditional context.

Definition 3.2 If $\Gamma$ and $\Delta$ are sets of second-order formulas, then $\Gamma \rightarrow \Delta$ is called a sequent. The conditional validity of a sequent $\Gamma \rightarrow \Delta$ with respect to a conditional assignment $\beta$ is defined by

$$
\left(\cup_{\psi \in \Gamma}[\neg \psi]^{\beta}\right) \cup\left(\cup_{\psi \in \Delta}[\psi]^{\beta}\right)
$$

and $\Gamma \rightarrow \Delta$ is said to be correct if and only if

$$
\left(\cup_{\psi \in \Gamma}[\neg \psi]^{\beta}\right) \cup\left(\cup_{\psi \in \Delta}[\psi]^{\beta}\right)=\Omega
$$

for all assignments $\beta$. An inference rule $R$ is a pair consisting of a finite sequence $\left(\Gamma_{1}, \Gamma_{2}, \ldots, \Gamma_{n}\right)$ of sequents and a single sequent $\Gamma$ written as $\frac{\Gamma_{1}, \Gamma_{2}, \ldots, \Gamma_{n}}{\Gamma}$, and it is 
said to be correct if and only if the correctness of $\Gamma$ follows from the correctness of $\Gamma_{1}, \Gamma_{2}, \ldots, \Gamma_{n}$.

We want to apply the inference rules for second-order logic given by Takeuti in [50, pages 9-10 and pages 135-136]. By Boolean arithmetic, one can directly check that all structural and logical rules are correct. We illustrate this for the first weakening rule:

$$
\frac{\Gamma \rightarrow \Delta}{\phi, \Gamma \rightarrow \Delta}
$$

For each assignment $\beta$, one has

$$
\left(\cup_{\psi \in \Gamma}[\neg \phi]^{\beta}\right) \cup\left(\cup_{\psi \in \Delta}[\psi]^{\beta}\right) \subset\left(\cup_{\psi \in \Gamma \cup\{\phi\}}[\neg \phi]^{\beta}\right) \cup\left(\cup_{\psi \in \Delta}[\psi]^{\beta}\right)
$$

which leads to the correctness of (3-1). By Proposition 3.1, the left universal quantification rule

$$
\frac{\phi(t), \Gamma \rightarrow \Delta}{\forall x \phi(x), \Gamma \rightarrow \Delta}
$$

where $t$ is a term, is correct. As for the correctness of the right universal quantification rule

$$
\frac{\Gamma \rightarrow \Delta, \phi(y)}{\Gamma \rightarrow \Delta, \forall x \phi(x)}
$$

where $y$ does not occur freely in $\Gamma \rightarrow \Delta, \forall x \phi(x)$, assume

$$
\cup_{\psi \in \Gamma}[\neg \psi]^{\beta} \cup \cup_{\psi \in \Delta}[\psi]^{\beta} \cup[\phi(y)]^{\beta}=\Omega
$$

for all conditional assignments and let $\beta$ be an arbitrary conditional assignment. We want to show that:

$$
\cup_{\psi \in \Gamma}[\neg \phi]^{\beta} \cup \cup_{\psi \in \Delta}[\psi]^{\beta} \cup \cap_{n \in L^{0}(\mathbb{N})}[\phi]^{\beta\left[\frac{n}{x}\right]}=\Omega
$$

By Proposition 3.1, there exists $n_{*} \in L^{0}(\mathbb{N})$ such that $\cap_{n \in L^{0}(\mathbb{N})}[\phi(x)]^{\beta\left[\frac{n}{x}\right]}=[\phi(x)]^{\beta\left[\frac{n_{*}}{x}\right]}$. Choose a $y$-variant $\beta^{\prime}$ of $\beta$ that maps $y$ to $n_{*}$. Then (3-3) follows from (3-2) since $y$ does not occur freely in $\Gamma \rightarrow \Delta, \forall x \phi(x)$, so that the first two sets of the union (3-3) remain unchanged. Analogously, one shows the first-order existential quantification inference rules. The second-order quantifier inference rules are only relevant for second-order variables, as predicate constants do not appear in our language. The inference rules for second-order quantification can hence be proved analogously to the corresponding first-order rules. Thus, we obtain:

Lemma 3.3 If $\frac{\Gamma_{1}, \ldots, \Gamma_{n}}{\Gamma}$ is any deduction rule of second-order sequent calculus and $\Gamma_{1}, \ldots, \Gamma_{n}$ are correct, then $\Gamma$ is correct. In particular, if all elements of $\Gamma$ hold in $\mathcal{S}$ 
with truth value $\Omega$ and $\Gamma \rightarrow \phi$ is derivable with the rules of sequent calculus, then $\phi$ holds in $\mathcal{S}$ with truth value $\Omega$.

Theorem 3.4 All the axioms of $A C A_{0}$ attain the value $\Omega$ in the structure $\mathcal{S}$ for all conditional assignments.

Proof Let $\beta$ be an arbitrary conditional assignment. The verification of the basic axioms (see Simpson [47, page 4]) is immediate from the definitions. For the sake of completeness, we provide the elementary arguments below.

- $[\neg(x+1=0)]^{\beta}=\left([x+1=0]^{\beta}\right)^{c}=\{\omega: \beta(x)(\omega)+1=0\}^{c}=\emptyset^{c}=\Omega$.

- Since $\{\omega: \beta(x)(\omega)+1=\beta(y)(\omega)+1\}=\{\omega: \beta(x)(\omega)=\beta(y)(\omega)\}$, it follows from $[x+1=y+1]^{\beta}=\Omega$ that $[x=y]^{\beta}=\Omega$.

- Similarly, one can verify that $[x+0=x]^{\beta}=\Omega,[x+(y+1)=(x+y)+1]^{\beta}=\Omega$, $[x \cdot 0=0]^{\beta}=\Omega,[x \cdot(y+1)=(x \cdot y)+x]^{\beta}=\Omega$, and $[\neg(x<0)]^{\beta}=\Omega$.

- $[x<y+1]^{\beta}=\Omega$ is equivalent to $\{\omega: \beta(x)(\omega)=\beta(y)(\omega)\} \cup\{\omega: \beta(x)(\omega)<$ $\beta(y)(\omega)\}=\Omega$, which means that $[(x<y) \vee(x=y)]^{\beta}=\Omega$.

As for the second-order induction scheme, we have to verify that:

$$
[(0 \in X \wedge \forall x(x \in X \rightarrow x+1 \in X)) \rightarrow \forall x(x \in X)]^{\beta}=\Omega
$$

By conditionally evaluating the previous formula and rewriting it by using Boolean arithmetic, we must verify that $A \subset B$, where:

$$
\begin{aligned}
& A:=i(0, \beta(X)) \cap \bigcap_{n \in L^{0}(\mathbb{N})}\left(\left([i(x, X)]^{\beta\left[\frac{n}{x}\right]}\right)^{c} \cup[i(x+1, X)]^{\beta\left[\frac{n}{x}\right]}\right) \\
& B:=\bigcap_{n \in L^{0}(\mathbb{N})}[i(x, X)]^{\beta\left[\frac{n}{x}\right]}
\end{aligned}
$$

But this is immediate from the stability of $\beta(X)$.

Finally, we verify the arithmetical comprehension scheme, that is, we want to show that

$$
[\exists X \forall x(x \in X \leftrightarrow \phi(x))]^{\beta}=\Omega
$$

for any arithmetical ${ }^{4}$ formula $\phi(x)$ in which $X$ does not occur freely. By Proposition 3.1, $A_{\phi}:=\cup_{n \in L^{0}(\mathbb{N})}[\phi(x)]^{\beta\left[\frac{n}{x}\right]}$ is attained. Suppose for a moment that

$$
N_{\phi}:=\left\{n \in L^{0}(\mathbb{N}):[\phi(x)]^{\beta\left[\frac{n}{x}\right]}=A_{\phi}\right\}
$$

\footnotetext{
${ }^{4}$ Recall that a formula of $L_{2}$ is said to be arithmetical if it contains no set quantifiers, see eg Simpson [47].
} 
satisfies stability. Then

$$
[\phi(x)]^{\beta\left[\frac{n}{x}\right]}=i\left(n, N_{\phi} \mid A_{\phi}\right)
$$

for all $n \in L^{0}(\mathbb{N})$. Indeed, for $n_{0} \in L^{0}(\mathbb{N})$, let $n_{1} \in N_{\phi}$ be such that $n_{0}\left|B=n_{1}\right| B$ where $B=i\left(n_{0}, N_{\phi} \mid A_{\phi}\right)$, and put $n_{2}=n_{0}\left|B+n_{1}\right| B^{c}$. By stability of $N_{\phi}$, we have:

$$
[\phi(x)]^{\beta\left[\frac{n_{0}}{x}\right]}=[\phi(x)]^{\beta\left[\frac{n_{2}}{x}\right]} \cap\left[n_{2}=n_{0}\right]^{\beta}=A_{\phi} \cap B=B
$$

By Boolean arithmetic, it follows from (3-4) that

$$
[\forall x(x \in X \leftrightarrow \phi(x))]^{\beta\left[\frac{Y_{\phi} \mid A_{\phi}}{X}\right]}=\Omega
$$

which proves comprehension. Thus it remains to verify that $N_{\phi}$ is stable under concatenations for all formulas $\phi$, which we will prove by an induction on arithmetical formulas. First, since addition and multiplication commute with concatenations ${ }^{5}$, for any first-order term $t=t(x)$, by an induction on terms, one has

$$
[t(x)]^{\beta\left[\frac{\sum_{k} n_{k} \mid A_{k}}{x}\right]}=\sum_{k}[t(x)]^{\beta\left[\frac{n_{k}}{x}\right]} \mid A_{k}
$$

for all measurable partitions $\left(A_{k}\right)$ and every countable family $\left(n_{k}\right)$ in $L^{0}(\mathbb{N})$. Since also order and concatenations commute and due to (2-3), $N_{\phi}$ is stable for all atomic formulas $\phi$.

Let $\phi$ and $\psi$ be two arithmetical formulas such that $N_{\phi}\left|A_{\phi}, N_{\psi}\right| A_{\psi} \in \mathcal{P}$. Then we have:

$$
\begin{aligned}
{[(\phi \wedge \psi)(x)]^{\beta\left[\frac{n}{x}\right]} } & =[\phi(x)]^{\beta\left[\frac{n}{x}\right]} \cap[\psi(x)]^{\beta\left[\frac{n}{x}\right]} \\
& =i\left(n, N_{\phi} \mid A_{\phi}\right) \cap i\left(n, N_{\psi} \mid A_{\psi}\right) \\
& =i\left(n, N_{\phi}\left|A_{\phi} \sqcap N_{\psi}\right| A_{\psi}\right)
\end{aligned}
$$

Moreover, for a negation one obtains:

$$
[\neg \phi(x)]^{\beta\left[\frac{n}{x}\right]}=i\left(n, N_{\phi} \mid A_{\phi}\right)^{c}=i\left(n,\left(N_{\phi} \mid A_{\phi}\right)^{\sqsubset}\right)
$$

Finally, let $\theta(x, y)$ be arithmetical and $\phi(y)=\exists x \theta(x, y)$. Clearly, $A_{\phi}=A_{\theta}$. By the established, we can already define a pairing function, product of stable sets and their projections, see eg Simpson [47, pages 66-69]. The pairing function $(i, j) \mapsto(i+j)^{2}+i$ underlying the definition of a product commutes with concatenations since this is the case for addition and multiplication. Whence if

$$
N_{\theta}=\left\{(n, m) \in L^{0}(\mathbb{N})^{2}:[\theta(x, y)]^{\beta\left[\frac{n}{x}, \frac{m}{y}\right]}=A_{\theta}\right\}
$$

\footnotetext{
${ }^{5}$ That is, $\sum_{k} n_{k}\left|A_{k} \circ \sum_{k} m_{k}\right| B_{k}=\sum_{k, h}\left(n_{k} \circ m_{h}\right) \mid A_{k} \cap B_{h}$ for $\circ \in\{+, \cdot\}$.
} 
is stable, then its projection to the first coordinate is stable, and by definition equal to $N_{\phi}$.

We now state the main theorem of this section.

Theorem 3.5 If $\phi$ is a consequence of $A C A_{0}$, then $\phi$ holds in $\mathcal{S}$ with truth value $\Omega$.

Proof By Theorem 3.4, all axioms of $\mathrm{ACA}_{0}$ have truth value $\Omega$ in $\mathcal{S}$. By Lemma 3.3, truth value $\Omega$ is preserved under the rules of second-order sequent calculus. Thus, the theorem follows.

\section{Harvesting the fruits}

A first step towards applications of the model-theoretic results in this article is to investigate connections between a conditional and a standard model. An aim of this section is to understand consequences of the transfer principle and interpret them properly in a standard setting. For example, the structure $L^{0}(\mathbb{N})$ are the natural numbers in the 'conditional model', while interpreted in a standard model, it is the space of $\mathbb{N}$-valued measurable functions on a $\sigma$-finite measure space. We shall discover connections to different parts of analysis such as set-valued analysis and measurable selection theory, vector-valued analysis and probabilistic analysis with applications to areas such as stochastic control, vector optimization and mathematical economics. An important observation is that application of theorems in a 'conditional model' preserves measurability systematically by construction. Moreover, we will demonstrate that a transfer principle unifies a conditional version of classical theorems which were proved by hand previously. Finally, new examples of theorems and applications shall underline the usefulness of a transfer principle.

\subsection{Set theory}

We begin by collecting basic set-theoretical vocabulary in a conditional setting. The inclusion relation (see Simpson [47, Definition II.3.1]) on the conditional power set $\mathcal{P}$ can be interpreted by the relation

$$
N|A \sqsubseteq M| B \quad \text { if and only if } A \subseteq B \text { and } N|A \subseteq M| A .
$$

This relation coincides with the conditional inclusion relation in conditional set theory, see Drapeau et al [15, Definition 2.8] for the abstract formulation in the context of an 
arbitrary complete Boolean algebra, and see Jamneshan et al [36, Definition 2.5] for a formulation in the context of an associated measure algebra.

The product of two sets $N|A, M| B \in \mathcal{P}$ is the set

$N \times M \mid A \cap B$

by interpreting [47, Definition II.3.1] in $\mathcal{S}$. This definition extends the definition of a conditional product in conditional set theory [15, Definition 2.14]. Indeed, a conditional product was introduced in [15] only for sets of the form $N=N \mid \Omega$ (if we consider the setting of the present paper). It was realized in [36] that the definition given in [15] does not suffice for proving a conditional version of Fubini's theorem. The extended definition then used in [36, Section 5.1] coincides with (4-2).

Following [47, Definition II.3.1], a function $f: N|A \rightarrow M| B$ can be interpreted as a subset $W|C \sqsubseteq N \times M| A \cap B$ such that for each $n|C \in N| C$ there is a unique $m|C \in M| C$ such that $(n, m)|C \in W| C$. This definition extends the one of a conditional function in [15, Definition 2.17] (similarly as the definition of a product (4-2) extends the definition of a conditional product in [15]). The concept of a conditional function is a significant abstraction of earlier concepts named a regular function in Detlefsen and Scandolo [12, Definition 4, Proposition 1], a stable function in Cheridito et al [10, Definition 4.2], and a local function in Filipovic et al [19, Definition 3.1]. The characteristic property of a conditional function is that evaluation of its values commutes with concatenations.

From [47, Lemma II.2.1] we know that $\left(L^{0}(\mathbb{N}),+, \cdot, 0,1,<\right)$ is a commutative ordered semiring with cancellation. For instance, the totality property $m<n \vee m=n \vee n<m$ reads as the statement that for each pair $n, m \in L^{0}(\mathbb{N})$ there is a partition $(\{m<n\},\{m=$ $n\},\{n<m\})$. This interpretation coincides with the definition of conditionally total in [15, Definition 2.15].

A set $N \mid A$ is said to be finite whenever there exists $k \in L^{0}(\mathbb{N})$ such that $n|A<k| A$ for all $n|A \in N| A$, see [47, page 67], which coincides with the notion of conditionally finite, see [15, Definition 2.23]. In general, a finite set in the model $\mathcal{S}$ is not finite in a standard sense, it might even be uncountable from the latter perspective. However, for every finite set $N \mid A$ there exist a partition $\left(A_{k}\right)$ and a countable family $\left(N_{k}\right)$ of finite subsets of $\mathbb{N}$ such that $N\left|A=\left(\sum_{k} N_{k} \mid A_{k}\right)\right| A$, where we identified $N_{k}$ with the set of all measurable $n: \Omega \rightarrow N_{k}$, cf [15, Lemma 2.22]. The collection of all finite sequences of length $n \in L^{0}(\mathbb{N})$ is the set of functions $\{1 \leqslant m \leqslant n\} \rightarrow L^{0}(\mathbb{N})$, see [47, Definition II.3.3], which is precisely the construction given in the paragraph after [15, Definition $2.20]$.

A sequence in a set $N$ is a function $f: L^{0}(\mathbb{N}) \rightarrow N$, compare with the definition of a conditional sequence in [15, Example 2.2.1]. From a standard point of view such a 
sequence is a net which is parametrized by $L^{0}(\mathbb{N})$ and commutes with concatenations, cf Drapeau et al [17, Section 2].

Let $\left(N_{k} \mid A_{k}\right)$ be a sequence in the power set $\mathcal{P}$. Using the element relation (see Definition 2.2), we see that the intersection of $\left(N_{k} \mid A_{k}\right)$ can be identified with

$$
\sqcap_{k} N_{k}\left|A_{k}:=V\right| B
$$

where:

$$
\begin{aligned}
& B:=\cup\left\{B^{\prime} \in \mathcal{F}: B^{\prime} \subset \cap_{k} A_{k}, \cap_{k}\left(N_{k} \mid B^{\prime}\right) \neq \emptyset\right\} \\
& V:=\left\{n \in L^{0}(\mathbb{N}): n \mid B \in \cap_{k}\left(N_{k} \mid B\right)\right\}
\end{aligned}
$$

and that the union of $\left(N_{k} \mid A_{k}\right)$ can be identified with

$$
\sqcup_{k} N_{k}\left|A_{k}:=W\right| C
$$

where:

$$
\begin{aligned}
C & :=\cup_{k} A_{k} \\
W & :=\left\{n \in L^{0}(\mathbb{N}): \cup_{k}\left(i\left(n, N_{k}\right) \cap A_{k}\right)=C\right\}
\end{aligned}
$$

The complement of a set was defined in (2-2). We have seen that the set operations in $\mathcal{S}$ recover the conditional set operations [15, page 567]. In [15, Corollary 2.10], it was proved that the conditional power set has the structure of a complete Boolean algebra, which is a fundamental result for basic constructions in conditional topology [15, Section 3 ] and conditional measure theory [36]. The transfer principle for $\mathrm{ACA}_{0}$ implies a weaker statement, namely that the power set $\mathcal{P}$ has the structure of a $\sigma$-complete Boolean algebra, see [47].

\subsection{Real analysis and linear algebra}

A detailed construction of the conditional real numbers and their conditional algebraic, order and topological properties are developed in Jamneshan [34, Chapter 5] where all properties are proved from conditional set theory. We will argue that most of these properties and related results are consequences of the transfer principle.

In the 'conditional model' $\mathcal{S}$, the integers (see Simpson [47, page 73]) are the space $L^{0}(\mathbb{Z})$ of measurable integer-valued functions. From [47, Theorem II.4.1] we know that $L^{0}(\mathbb{Z})$ is an Euclidean ordered integral domain. Similarly, the space $L^{0}(\mathbb{Q})$ of measurable rational-valued functions are the rational numbers in $\mathcal{S}$. By [47, Theorem II.4.2], $L^{0}(\mathbb{Q})$ is an ordered field; compare this with the conditionally ordered field 
of conditional rational numbers in [15, Example 4.2.1]. The topological completion of $L^{0}(\mathbb{Q})$ inside $\mathcal{S}$ are the real numbers which by a standard approximation argument can be identified with the space $L^{0}(\mathbb{R})$ of real-valued measurable functions, see [47, Definition II.4.4]. Now [47, Theorem II.4.5] implies that $L^{0}(\mathbb{R})$ is an Archimedean ordered field, cf [34, Lemma 5.2.12 and Theorem 5.2.7].

Notice that the absolute value of real numbers maps to $L_{+}^{0}(\mathbb{R}):=\left\{x \in L^{0}(\mathbb{R}): x \geqslant 0\right\}$. Let $L_{++}^{0}(\mathbb{Q}):=\left\{x \in L^{0}(\mathbb{Q}): x>0\right\}$. An open ball in $L^{0}(\mathbb{R})$ is a set

$$
B(q, r):=\left\{x \in L^{0}(\mathbb{R}):|x-q|<r\right\}
$$

where $q \in L^{0}(\mathbb{Q})$ and $r \in L_{++}^{0}(\mathbb{Q})$, see [47, page 81]. An open set $O$ is the union of a sequence of open balls $\sqcup_{k} B\left(q_{k}, r_{k}\right)$, see [47, Definition II.5.6]. A closed set is the complement of an open set, see [47, Definition II.5.12]. For example, for any $r \in L_{++}^{0}(\mathbb{Q})$, the set $\left\{x \in L^{0}(\mathbb{R}):|x| \leqslant r\right\}$ is closed, but the standard complement of $B(0, r)$ is in general not. However, it can be easily verified that every $\mathcal{S}$-closed set is sequentially closed, ie it contains the limit of any almost everywhere converging standard sequences.

Remark 4.3 The Euclidean topology of the real numbers $L^{0}(\mathbb{R})$ in $\mathcal{S}$ is the order topology in a standard model which renders pointwise addition and multiplication continuous. Thus, $L^{0}(\mathbb{R})$ becomes a topological algebra, in particular a topological module of rank 1 over itself. This fact is exploited in Filipovic et al [19], Cheridito et al [10], Drapeau et al [15], and Jamneshan and Zapata [38] to build a functional analytic discourse in $L^{0}(\mathbb{R})$-modules. We shall observe later that a functional analytic discourse in $L^{0}(\mathbb{R})$-modules is the reflection of classical functional analysis, albeit in a 'conditional model'. So far, we know that $L^{0}(\mathbb{R})$ is 1 -dimensional Euclidean space in the 'conditional model' $\mathcal{S}$.

Let $L^{0}(\mathbb{R})^{n}$ denote the set of finite sequences $\{1 \leqslant m \leqslant n\} \rightarrow L^{0}(\mathbb{R})$. Write $n$ as $\sum_{k} n_{k} \mid A_{k}$ for $\left(n_{k}\right)$ in $\mathbb{N}$ and $\left(A_{k}\right)$ a measurable partition. Then one can view $L^{0}(\mathbb{R})^{n}$ as $\sum_{k} L^{0}(\mathbb{R})^{n_{k}} \mid A_{k}$ where each $L^{0}(\mathbb{R})^{n_{k}}$ is a standard product. The absolute value extends from $L^{0}(\mathbb{R})$ to an Euclidean norm on $L^{0}(\mathbb{R})^{n}$ which for $x=\sum_{k}\left(x_{1}, \ldots, x_{n_{k}}\right) \mid A_{k}$ is defined by:

$$
\|x\|:=\sum_{k} \sqrt{x_{1}^{2}+\ldots+x_{n_{k}}^{2}} \mid A_{k}
$$

Convergence in the conditional Euclidean space $L^{0}(\mathbb{R})^{n}$ can be characterized by almost everywhere convergence. Suppose that $n \in \mathbb{N}$ (the general case $n=\sum_{k} n_{k} \mid A_{k}$ follows 
by localizing the subsequent construction to each $A_{k}$ and gluing). Let $\left(x_{k}\right)$ be a standard sequence in $L^{0}(\mathbb{R})^{n}$ converging almost everywhere to $x$. Given $r \in L_{++}^{0}(\mathbb{Q})$, let:

$$
k_{r}(\omega)=\inf \left\{k^{\prime} \in \mathbb{N}:\left\|x_{m}-x\right\|(\omega)<r(\omega) \text { for all } m \geqslant k^{\prime}\right\}
$$

Notice that $k_{r}$ is measurable. Obtain from $\left(x_{k}\right)$ the conditional sequence $x_{k}:=\sum_{n} x_{k_{n}} \mid A_{n}$, $k=\sum_{n} k_{n} \mid A_{n}$. By construction, $\left\|x_{k}-x\right\|<r$ for all $k \geqslant k_{r}$. Conversely, it is easy to see that if $\left(x_{k}\right)_{k \in L^{0}(\mathbb{N})}$ is a conditional sequence conditionally converging to $x$, then the standard subnet $\left(x_{k}\right)_{k \in \mathbb{N}}$ resulting by embedding $\mathbb{N}$ into $L^{0}(\mathbb{N})$ via $n \mapsto n 1_{\Omega}$ converges to $x$ almost everywhere.

Remark 4.4 The $\mathcal{S}$-Euclidean norm (4-5) is an example of $L^{0}(\mathbb{R})$-valued vector norms which appear in different parts of analysis such as the analysis of Lebesgue-Bochner spaces and vector integration (see eg Diestel and Uhl [13], Haydon et al [32], and Hytönen et al [33]), financial modeling (see eg Hansen and Richard [31], Filipovic et al [20], and Cheridito et al [8]), or probabilistic analysis (see eg Guo [27] and Haydon et al [32]). In Section 4.20, we will continue to discuss the connection of $L^{0}(\mathbb{R})$-valued vector normed spaces and Banach spaces in the 'conditional model' $\mathcal{S}$.

By the transfer principle, we obtain from [47, Lemma III.2.1] a Bolzano-Weierstraß theorem in $\mathcal{S}$. Recall that a sequence $\left(x_{k}\right)$ in $L^{0}(\mathbb{R})^{n}, n \in L^{0}(\mathbb{N})$, is said to be bounded if there exists $r \in L_{++}^{0}(\mathbb{Q})$ such that $\left\|x_{k}\right\|<r$ for all $k \in L^{0}(\mathbb{N})$.

Theorem 4.5 Let $\left(x_{k}\right)$ be a bounded sequence in $L^{0}(\mathbb{R})^{n}$. Then $x=\lim \sup _{k} x_{k}$ exists. Moreover, there exists a subsequence $\left(x_{k_{p}}\right)$ converging to $x$.

The previous statement is reminiscent of a 'measurable' or 'conditional' version of the Bolzano-Weierstraß theorem as proved in Kabanov and Stricker [39, Lemma 2], in Föllmer and Schied [21, Lemma 1.64] and in Cheridito et al [10, Theorem 3.8] respectively. Indeed, any standard sequence $\left(x_{k}\right)$ in $L^{0}(\mathbb{R})$ can be extended to a conditional sequence (see above). If $\left(x_{k}\right)$ is bounded ( $\left.\sup _{k}\left|x_{k}\right|<\infty\right)$, then there is a conditional subsequence $\left(x_{k_{p}}\right)$ which converges to $x=\lim \sup _{k} x_{k}$ by Theorem 4.5. Choosing the standard subsequence $\left(x_{k_{p}}\right)_{p \in \mathbb{N}}$ in the net $\left(x_{k_{p}}\right)_{p \in L^{0}(\mathbb{N})}$, we conclude $x_{k_{p}} \rightarrow x$ almost everywhere.

Compactness within $\mathrm{ACA}_{0}$ is introduced in [47, Definition III.2.3] as a form of sequential compactness. We have the following characterization.

Proposition 4.6 Let $W$ be a stable subset of $L^{0}(\mathbb{R})^{n}, n=\sum_{k} n_{k} \mid A_{k} \in L^{0}(\mathbb{N})$. Then the following are equivalent. 
(i) $W$ is conditionally compact.

(ii) Heine-Borel property: For every conditional sequence $\left(O_{i}\right)$ of open sets such that $W \sqsubseteq \sqcup_{i} O_{i}$ there exists a conditionally finite subsequence $\left(O_{i_{j}}\right)$ such that $W \sqsubseteq \sqcup_{j} O_{i_{j}}$.

(iii) $W$ can be represented as $\sum_{k} W_{k} \mid A_{k}$ where each $W_{k}$ is the set of almost everywhere selections of an Effros measurable compact-valued map in $\mathbb{R}^{n_{k}}$.

Proof The equivalence of (i) and (ii) follows from [47, Theorem IV.1.5] and the transfer principle 3.5. The equivalence of (ii) and (iii) is proved in Jamneshan and Zapata [38, Section 5].

Remark 4.7 The notion of conditional compactness was introduced in Drapeau et al [15, Definition 3.24]. A conditional version of the Heine-Borel theorem for general conditional metric spaces was established in [15, Theorem 4.6]. A simple example of a conditionally compact set in $L^{0}(\mathbb{R})$ are finite unions of intervals

$$
[a, b]:=\left\{x \in L^{0}(\mathbb{R}): a \leqslant x \leqslant b\right\}
$$

where $a<b$ in $L^{0}(\mathbb{R})$.

The following minimum theorem was proved in [10, Theorem 4.4] in finite dimensions, and in full generality in Jamneshan and Zapata [38, Theorem 5.13], and applied in eg Cheridito et al [8] and Jamneshan et al [37] successfully to stochastic control.

Theorem 4.8 Let $W$ be a conditionally compact subset of $L^{0}(\mathbb{R})^{n}, n \in L^{0}(\mathbb{N})$, and let $f: W \rightarrow L^{0}(\mathbb{R})$ be a conditionally lower semi-continuous function. Then $f$ has a minimum.

Proof The proof can be done in $\mathrm{WKL}_{0}$, which is a subsystem of $\mathrm{ACA}_{0}$, using the Heine-Borel covering property [47]. The claim then follows from Theorem 3.5.

We can derive the following variant of the previous theorem in set-valued analysis an important aspect of which is the avoidance of measurable selection arguments.

Corollary 4.9 Let $f: \Omega \times \mathbb{R}^{n} \rightarrow \mathbb{R}$ be a normal integrand ${ }^{7}$, and let $X: \Omega \rightarrow 2^{\mathbb{R}^{n}}$ be an Effros measurable compact-valued map. Then there exists a measurable function $x: \Omega \rightarrow \mathbb{R}^{n}$ such that $f(\omega, x(\omega))=\min _{x \in X(\omega)} f(\omega, x)$ almost everywhere.

${ }^{6}$ A set-valued function $X: \Omega \rightarrow 2^{\mathbb{R}^{n}}$ is said to be Effros measurable, if $\{\omega: X(\omega) \cap O \neq$ $\emptyset\} \in \mathcal{F}$ for all open sets $O$ in $\mathbb{R}^{n}$.

${ }^{7} \mathrm{~A}$ function $f: \Omega \times \mathbb{R}^{n} \rightarrow \mathbb{R}$ is said to be a normal integrand if its graph is Effros measurable and closed-valued. 
Proof By proposition 4.6, one can identify $X$ with a conditionally compact set in $L^{0}(\mathbb{R})^{n}$. From a result in Jamneshan et al [37, Section 5], one can identify $f$ with a sequentially lower semi-continuous ${ }^{8}$ function $L^{0}(\mathbb{R})^{n} \rightarrow L^{0}(\mathbb{R})$. Theorem 4.8 proves the claim.

A notion of an $L^{0}(\mathbb{R})$-derivative for functions $f: L^{0}(\mathbb{R})^{n} \rightarrow L^{0}(\mathbb{R})$ is introduced in Cheridito et al [10, Section 7]. An interpretation of the definition of a derivative in second-order arithmetic yields the same concept which is defined below for $n=1$.

Definition 4.10 Let $f: L^{0}(\mathbb{R}) \rightarrow L^{0}(\mathbb{R})$ be a stable function such that $f\left(x_{k}\right) \rightarrow f(x)$ almost everywhere whenever $x_{k} \rightarrow x$ almost everywhere. The value

$$
f^{\prime}(x)=\lim _{h \rightarrow 0} \frac{f(x-h)-f(x)}{h}
$$

is called the conditional derivative of $f$ at $x$ if it exists.

The following conditional version of the Peano existence theorem, derived from [47, Theorem IV.8.1], has not been proved previously. It can be applied to solve random ordinary differential equations.

Theorem 4.11 Let $a, b \in L^{0}(\mathbb{R})$ with $a, b>0$. Let $f:[-a, a] \times[-b, b] \rightarrow L^{0}(\mathbb{R})$ be stable sequentially continuous. Then the random ordinary differential equation

$$
\frac{d y}{d x}=f(x, y), \quad y(0)=0,
$$

has a sequentially continuous differentiable solution $y=\phi(x)$ on the interval $-\alpha \leqslant$ $x \leqslant \alpha$ where $\alpha=\min (a, b / M)$ and

$$
M=\max \{|f(x, y)|:-a \leqslant x \leqslant a,-b \leqslant y \leqslant b\}
$$

In [10, Section 2], some basic results in linear algebra are extended to the space $L^{0}(\mathbb{R})^{n}$ which culminates in a conditional version of the orthogonal decomposition theorem $[10$, Corollary 2.12], whereby $L^{0}(\mathbb{R})^{n}$ is viewed as an module of rank $n$ over the commutative ring $L^{0}(\mathbb{R})$. By basic linear algebra in second-order arithmetic [47], $L^{0}(\mathbb{R})^{n}$ is a vector space of dimension $n$ in $\mathcal{S}$, and all results in [10, Section 2] are consequences of the transfer principle. Actually, they directly extend to the case where $n(\omega)$ is a measurable dimension.

\footnotetext{
${ }^{8}$ Lower semi-continuity in $\mathcal{S}$ can be interpreted as sequential lower semi-continuity in a standard model, ie $f(x) \leqslant \liminf f\left(x_{k}\right)$ almost everywhere whenever $\left(x_{k}\right)$ converges almost everywhere to $x$, see the discussion above relating convergence in the $\mathcal{S}$-Euclidean topology with almost everywhere convergence.
} 


\subsection{Metric spaces}

In the axiomatic system of second-order arithmetic, the only definable metric spaces are separable and complete ones which are coded as a completion of a countable set with a prescribed rate of convergence, see Simpson [47, Definition II.5.1]. We characterize a complete and separable metric space in the conditional model $\mathcal{S}$ as a vector metric space in a standard model as follows.

Definition 4.13 A non-empty set $H$ is said to be a conditional set ${ }^{9}$ if there exists a restriction operation $\mid$ such that for every sequence $\left(x_{k}\right)$ in $H$ and every measurable partition $\left(A_{k}\right)$ there exists a unique element $x \in H$ such $x_{k}\left|A_{k}=x\right| A_{k}$ for all $k$. We name this unique element a concatenation and denote it by $x=\sum_{k} x_{k} \mid A_{k}$.

Let $H$ be a conditional set. A function $d: H \times H \rightarrow L_{+}^{0}(\mathbb{R})$ is a conditional metric if:

- $d\left(\sum_{k}\left(x_{k}, y_{k}\right) \mid A_{k}\right)=\sum_{k} d\left(x_{k}, y_{k}\right) \mid A_{k}$ for all sequences $\left(x_{k}, y_{k}\right)$ in $H \times H$ and measurable partitions $\left(A_{k}\right)$

- $d(x, y)=0$ if and only if $x=y$

- $d(x, y)=d(y, x)$ for all $x, y \in H$

- $d(x, z) \leqslant d(x, y)+d(y, z)$ for all $x, y, z \in H$

A conditional metric space $H$ is said to be

- conditionally separable, if there exists a countable set $G \subset H$ such that for all $x \in H$ there is a sequence $\left(x_{k}\right)$ in $G$ and $n_{1}<n_{2}<\ldots$ in $L^{0}(\mathbb{N})$ such that $d\left(x_{n_{m}}, x\right) \rightarrow 0$ almost everywhere where $x_{n}:=\sum_{k} x_{k} \mid\{n=k\}$ for $n \in L^{0}(\mathbb{N})$; and

- conditionally complete, if for every conditional Cauchy sequence ${ }^{10}\left(x_{k}\right)$ there exists $x$ such that $d\left(x_{k}, x\right) \rightarrow 0$ almost everywhere.

A conditional metric space is said to be conditionally Polish, if it is conditionally separable and conditionally complete.

Examples 4.14 a) Let $(E, d)$ be a separable metric space with countable dense subset $S$. The metric $d$ extends to a vector metric on $L^{0}(E)$ with values in $L_{+}^{0}(\mathbb{R})$ via $d(x, y)(\omega):=d(x(\omega), y(\omega))$ almost everywhere. Let $G$ be the set of all constant functions with values in $S$. See Drapeau et al [16, Sections 2 and 4] for a proof that $L^{0}(E)$ is conditionally complete.

\footnotetext{
${ }^{9}$ See [15, Definition 2.1] for a formal definition.

${ }^{10}$ That is, a sequence $\left(x_{k}\right)$ such that for all $r \in L_{++}^{0}(\mathbb{Q})$ there exists $n \in L^{0}(\mathbb{N})$ with $d\left(x_{m}, x_{p}\right)<r$ for all $m, p \in L^{0}(\mathbb{N})$ with $m, p \geqslant n$.
} 
b) Suppose that $(\Omega, \mathcal{F}, \mathbb{P})$ is a standard Borel probability space, and let $\mathcal{G} \subset \mathcal{F}$ be a sub- $\sigma$-algebra. For a non-negative random variable $x: \Omega \rightarrow \mathbb{R}$, define the extended conditional expectation $\mathbb{E}[x \mid \mathcal{G}]:=\lim _{n \rightarrow \infty} \mathbb{E}[x \wedge n \mid \mathcal{G}]$. For a real number $p \in[1, \infty)$, let $L^{p}(\mathcal{F} \mid \mathcal{G})$ denote the space of $\mathcal{F}$-measurable random variables such that $\mathbb{E}\left[|x|^{p} \mid \mathcal{G}\right]<\infty$ almost everywhere. The space $L^{p}(\mathcal{F} \mid \mathcal{G})$ can be represented in 'tensorial' form as $L^{0}(\mathcal{G}, \mathbb{R}) \cdot L^{p}(\mathcal{F}, \mathbb{R})$ within the space $L^{0}(\mathcal{F}, \mathbb{R})$, see $\left[6\right.$, Proposition 1]. The mapping $x \mapsto\left(\mathbb{E}\left[|x|^{p} \mid \mathcal{G}\right]\right)^{1 / p}$ defines a $\mathcal{G}$-conditional norm on $L^{p}(\mathcal{F} \mid \mathcal{G})$, ie a vector norm with values in $L_{+}^{0}(\mathcal{G}, \mathbb{R})$ which equips $L^{p}(\mathcal{F} \mid \mathcal{G})$ with the structure of a topological $L^{0}(\mathcal{G}, \mathbb{R})$-module, see the discussion in Filipovic et al [19, Example 2.5] and Cerreia-Vioglio et al [6, Section 8]. The induced $\mathcal{G}$-stable vector metric is given by $d(x, y):=\mathbb{E}\left[|x-y|^{p} \mid \mathcal{G}\right]$. Let $S$ be a countable dense in $L^{p}(\mathcal{F}, \mathbb{R})$, and let $G$ be the space of all products $x y$ where $x$ is a constant rational-valued random variable and $y \in S$. Conditional completeness follows from Vogelpoth [51, Theorem 3.2.3]. Notice that here the underlying measure space is $(\Omega, \mathcal{G}, \mathbb{P})$.

c) Let $E$ be a standard Polish space, and let $F: \Omega \rightrightarrows E$ be an Effros measurable and closed-valued map. Then the set of almost everywhere selections of $F$ have the structure of a conditional Polish space.

Remark 4.15 A conditional metric induces a standard topology on the set $H$ given by a base $\left\{B(x, r): x \in H, r \in L_{+}^{0}(\mathbb{Q})\right\}$. This standard topology on $H$ is in general not $\mathbb{R}$-metrizable. For several basic topological notions such as continuity and convergence, we have equivalences between their conditional and standard variants, see Drapeau et al [15, Section 3] for a systematic study.

We have the following classification result.

Proposition 4.16 Every conditional Polish space corresponds uniquely to a Polish space in the conditional model $\mathcal{S}$.

Proof By definition, every Polish space in $\mathcal{S}$ is a conditional Polish space in the standard sense of Definition 4.13 (one can change from a conditionally countable family to a standard countable family by considering only the constant-valued indices). Conversely, let $H$ be a conditional Polish space, and let $G \subset H$ be countable and conditionally dense. Then each $x \in H$ can be identified with a conditional sequence $\left(x_{n}\right)_{n \in L^{0}(\mathbb{N})}$ in the concatenation hull

$$
\operatorname{st}(G)=\left\{\sum_{k} x_{k} \mid A_{k}:\left(x_{k}\right) \text { in } G,\left(A_{k}\right) \text { measurable partition }\right\}
$$


such that $m<n$ implies $d\left(x_{m}, x_{n}\right)<2^{-m}$ for all $n, m \in L^{0}(\mathbb{N})$. Indeed, by a conditional version of the axiom of choice, see Drapeau et al [15, Theorem 2.26], one finds a conditional sequence $\left(x_{n}\right)_{n \in L^{0}(\mathbb{N})}$ such that $x_{n} \in O_{n}$ for all $n \in L^{0}(\mathbb{N})$ where:

$$
O_{n}=\left\{y \in \operatorname{st}(G): d(x, y)<1 / 2^{n+1}\right\}
$$

As $\operatorname{st}(G)$ is countable from the perspective of $\mathcal{S}$, one concludes that $H$ is a Polish space in the model $\mathcal{S}$.

We prove a characterization of closed sets in $\mathcal{S}$ which establishes a link to set-valued analysis.

Proposition 4.17 Let $E$ be a standard Polish space, and let $W \subset L^{0}(E)$ be stable ${ }^{11}$. Then the following are equivalent.

(i) $W$ is closed in $\mathcal{S}$.

(ii) $W$ is sequentially closed.

(iii) There exists an Effros measurable and closed-valued map $X: \Omega \rightarrow 2^{E}$ such that $W$ coincides with the set of almost everywhere selections of $X$.

Proof We prove (i) $\Rightarrow$ (ii).

Let $\left(x_{k}\right)$ be a sequence in $W$ such that $d\left(x_{k}, x\right) \rightarrow 0$ almost everywhere for some $x \in$ $L^{0}(E)$, and let $G$ be a countable dense set in $E$. By contradiction, if $\mu(i(x, W \sqsubset))>0$, then one finds a conditional ball $B(y, r)$ with center $y \in L^{0}(G)$ and radius $r \in L_{++}^{0}(\mathbb{Q})$ such that $\mu\left(i\left(x, B(y, r) \sqcap W^{\sqsubset}\right)\right)>0$. But then there is also some $k$ such that $\mu\left(i\left(x_{k}, W^{\sqsubset}\right)\right)>0$ which is the desired contradiction. Hence $\mu\left(i\left(x, W^{\complement}\right)\right)=0$, and therefore $x \in W$.

We prove (ii) $\Rightarrow$ (i).

Let:

$$
I:=\left\{(y, r) \in L^{0}(G) \times L_{++}^{0}(\mathbb{Q}): B(y, r) \cap W \neq \emptyset\right\}
$$

Notice that $I$ is conditionally countable. By a conditional version of the axiom of choice [15, Theorem 2.26], one finds a conditional sequence $\left(x_{i}\right)$ such that $x_{i} \in B(y, r) \cap W$ for each $i=(y, r) \in I$. Now $W$ coincides with the Polish space defined by the countable set $\left\{x_{i}\right\}$ in the model $\mathcal{S}$.

The equivalence of (ii) and (iii) is proved in Jamneshan and Zapata [38, Section 5].

\footnotetext{
${ }^{11}$ Stability here and else where always refers to stability respectively closedness with respect to countable concatenations.
} 
As a direct consequence of the transfer principle, one obtains a conditional version of the Baire category theorem (see Simpson, [47, Theorem II.5.8]), Urysohn's lemma [47, Lemma II.7.3], Tietze's extension theorem [47, Theorem II.7.5], the Ascoli lemma [47, Theorem III.2.8], the Heine-Borel theorem [47, Theorem IV.1.5], a choice principle for compact sets [47, Theorem IV.1.8], and Tychonoff's theorem [47, Theorem III.2.5]. A conditional version of these theorems except Urysohn's lemma and Tietze's extension theorem were proved by hand previously, see Jamneshan and Zapata [38] for references. We state a conditional version of Urysohn's lemma in standard language. Recall that $[0,1]$ denotes the set of all real-valued measurable functions $0 \leqslant x \leqslant 1$.

Theorem 4.18 Let $H$ be a conditional Polish space, and let $W_{0}$ and $W_{1}$ be stable and sequentially closed subsets of $H$ such that $W_{0} \sqcap W_{1}=\{*\}$. Then there exists a stable function $f: H \rightarrow[0,1]$ such that $f\left(x_{k}\right) \rightarrow f(x)$ almost everywhere whenever $x_{k} \rightarrow x$ almost everywhere and $\{\omega: f(x)(\omega)=j\}=i\left(x, W_{j}\right)$ for all $j=0,1$ and $x \in H$.

Heuristically, the previous theorem states that two random sets with values in a Polish space which only intersect on a negligible set can be separated by a random $\{0,1\}$ valued function $f$ in the sense that the largest measurable set on which a random element $x$ falls into the first/second set coincides almost surely with the measurable set $\{\omega: f(x)(\omega)=0 / 1\}$. A notable aspect about this 'randomized' Urysohn's lemma is that we do not leave the Borel measurable world without invoking a measurable selection argument, or in other words, measurability is a result of the construction.

Remark 4.19 Conditional metric spaces were introduced in full generality in Drapeau et al [15]. Conditional metric spaces were applied in Jamneshan et al [37] to stochastic control. It was argued there that they are a viable alternative to the established measurable selection techniques. Moreover, examples of problems were presented which can be solved with conditional analysis techniques which, however, are beyond the scope of applicability of measurable selection techniques due to topological restrictions.

\subsection{Banach space theory}

In this section, we develop basics of Banach spaces in $\mathcal{S}$, and connect it with functional analysis in $L^{0}(\mathbb{R})$-modules. An $L^{0}(\mathbb{R})$-module $H$ is a module over the commutative algebra $L^{0}(\mathbb{R})$. This yields a function from the underlying measure space $(\Omega, \mathcal{F}, \mu)$ to $H$ by scalar multiplication with indicator functions $1_{A} \cdot x, A \in \mathcal{F}$ and $x \in H^{12}$. This enables

\footnotetext{
${ }^{12}$ Actually, it is the measure algebra associated to $(\Omega, \mathcal{F}, \mu)$ which acts on $H$.
} 
us to formalize concatenations in $H$. An element $x \in H$ is said to be a concatenation of a sequence $\left(x_{k}\right)$ in $H$ and a measurable partition $\left(A_{k}\right)$, if $1_{A_{k}} \cdot x=1_{A_{k}} \cdot x_{k}$ for all $k$. We say that $H$ satisfies the countable concatenation property, if for every sequence $\left(x_{k}\right)$ in $H$ and each measurable partition $\left(A_{k}\right)$ there exists a unique concatenation in $H$. Throughout all $L^{0}(\mathbb{R})$-modules are assumed to satisfy the countable concatenation property.

Definition 4.21 Let $H$ be an $L^{0}(\mathbb{R})$-module. A function $\|\cdot\|: H \rightarrow L_{+}^{0}(\mathbb{R})$ is said to be a conditional norm, if:

- $\|x\|=0$ if and only if $x=0$

- $\|r x\|=|r|\|x\|$ for all $r \in L^{0}(\mathbb{R})$ and $x \in H$

- $\|x+y\| \leqslant\|x\|+\|y\|$ for all $x, y \in H$

Since $H$ satisfies the countable concatenation property, a conditional norm is a stable function. Then $d(x, y):=\|x-y\|$ gives $H$ the structure of a conditional metric space. A conditional norm induces a standard topology given by the base $B(x, r):=\{y \in$ $H:\|x-y\|<r\}, r \in L_{++}^{0}(\mathbb{Q})$ and $x \in H$. The generated topology renders addition and $L^{0}(\mathbb{R})$-scalar multiplication continuous where we consider on $L^{0}(\mathbb{R})$ its order topology, see Remark 4.4. From Proposition 4.16 we have:

Proposition 4.22 There is a one-to-one correspondence from the class of conditionally separable and conditionally complete $L^{0}(\mathbb{R})$-normed modules in a standard model and the class of separable Banach spaces ${ }^{13}$ in the model $\mathcal{S}$.

One obtains the following examples of Banach spaces in the model $\mathcal{S}$ (see examples 4.14 for details).

Examples 4.23 a) For $p \in[1, \infty)$, the conditional $L^{p}$-space $L^{p}(\mathcal{F} \mid \mathcal{G})$ with respect to a standard Borel probability space.

b) The Bochner space $L^{0}(E)$ of equivalence classes of strongly measurable functions with values in a separable normed vector space $E$.

As a consequence of the transfer principle, we obtain a conditional version of the Hahn-Banach extension and separation theorems (see Simpson [47, Theorems IV.9.3, X.2.1]), the Banach-Steinhaus theorem [47, Theorem II.10.8], the Banach-Alouglu theorem [47, Remark X.2.4], and the Krein-Šmulian theorem [47, Theorem X.2.7]

\footnotetext{
${ }^{13} \mathrm{~A}$ separable Banach space in second-order arithmetic is introduced in [47, Definition II.10.1].
} 
for conditionally separable Banach spaces. We spell out a conditional version of the Banach-Steinhaus theorem below. The following definition is a standard interpretation of [47, Definition II.10.5] in $\mathcal{S}$.

Definition 4.24 Let $H$ and $K$ be conditionally separable and conditionally complete $L^{0}(\mathbb{R})$-normed modules. A function $f: H \rightarrow K$ is said to be:

- conditionally linear, if $f(x+r y)=f(x)+r f(y)$ for all $x, y \in H$ and $r \in L^{0}(\mathbb{R})$

- conditionally bounded, if there exists $r \in L_{++}^{0}(\mathbb{Q})$ such that $\|f(x)\|_{K} \leqslant r\|x\|_{H}$ for all $x \in H$

Notice that any conditionally linear function is stable. One can deduce from [47, Theorem II.10.7] that a conditionally linear and conditionally bounded operator is sequentially continuous, ie $\left\|f\left(x_{k}\right)-f(x)\right\|_{K} \rightarrow 0$ almost everywhere whenever $\left\|x_{k}-x\right\|_{H} \rightarrow 0$ almost everywhere. A conditional version of the Banach-Steinhaus theorem is obtained by an application of the transfer principle to [47, Theorem II.10.8]. One could think of its application in random operator theory and random differential equations, see eg Skorohod [48] and Strand [49].

Theorem 4.25 Let $H$ and $K$ be conditionally separable and complete $L^{0}(\mathbb{R})$-normed modules, and let $\left(f_{n}\right)$ be a standard sequence of $L^{0}(\mathbb{R})$-linear and sequentially continuous functions $f_{n}: H \rightarrow K$. If for every $x \in H$ there exists $r \in L_{++}^{0}(\mathbb{Q})$ such that $\left\|f_{n}(x)\right\|_{K} \leqslant r$ for all $n$, then there exists $q \in L_{++}^{0}(\mathbb{Q})$ such that $\left\|f_{n}(x)\right\|_{K} \leqslant q\|x\|_{H}$ for all $x$ and every $n$.

Remark 4.26 Conditional extension and separation arguments are applied in Filipovic et al [20] and Frittelli and Maggis [24] in risk measure theory. A conditional FenchelMoreau theorem is applied in Drapeau et al [17] in vector duality. A conditional Riesz representation theorem is used in Drapeau and Jamneshan [14] in decision theory.

Remark 4.27 Separation and duality results in topological $L^{0}(\mathbb{R})$-modules are established in Guo et al [29] and Filipovic et al [19] with respect to two types of module topologies respectively, their connection is discussed in Guo [27], see also the discussion in Jamneshan and Zapata [38]. The standard topologies employed in [29], and with a different motivation in Haydon et al [32], are extensions of the topology of convergence in probability to $L^{0}(\mathbb{R})$-vector norms on general $L^{0}(\mathbb{R})$-modules. The class of such probabilistic topologies does not yield a comprehensive functional analytic discourse in $L^{0}(\mathbb{R})$-modules, see Jamneshan and Zapata [38]. 
The second type of topologies introduced in [19] proved to be more susceptible to a comprehensive functional analytic discourse for which strong evidence was provided in [15] by embedding $L^{0}(\mathbb{R})$-module theory in conditional set theory and conditional topology. Conditional topological vector spaces are introduced in [15, Section 5]. In Drapeau et al [16] conditional completions of standard metric spaces are constructed and their connection to Lebesgue-Bochner spaces is established. The above listed consequences of the transfer principle have been proved by hand previously, see [38] for an overview and references.

A school of Russian mathematicians, starting with Kantorovic, studied to which extent results in functional analysis remain true if the real numbers are replaced by a Dedekind complete vector lattice of which $L^{0}(\mathbb{R})$ is one example. These investigations were naturally connected to Boolean-valued models. We refer to Kusraev and Kutateladze [42] and Kutateladze [43] for an extensive overview of this tradition.

\subsection{Hilbert spaces}

In this subsection, we will elaborate on basic results in separable Hilbert spaces ${ }^{14}$ where our focus lies on the conditional $L^{2}$-space

$$
L^{2}(\mathcal{F} \mid \mathcal{G})=\left\{x \in L^{0}(\mathcal{F}, \mathbb{R}): \mathbb{E}\left[x^{2} \mid \mathcal{G}\right]<\infty\right\}
$$

where $(\Omega, \mathcal{F}, \mathbb{P})$ is a standard Borel probability space and $\mathcal{G} \subset \mathcal{F}$ is a sub- $\sigma$-algebra, see examples 4.14. The inner product in $L^{2}(\mathcal{F} \mid \mathcal{G})$ is defined by $\langle x, y\rangle:=\mathbb{E}[x y \mid \mathcal{G}]$. Recall that for $L^{2}(\mathcal{F} \mid \mathcal{G})$ the base space is $(\Omega, \mathcal{G}, \mathbb{P})$.

Definition 4.29 A sequence $\left(x_{n}\right)_{n \in L^{0}(\mathbb{N})}$ in $L^{2}(\mathcal{F} \mid \mathcal{G})$ is said to be orthonormal, if

- $\left\langle x_{n}, x_{m}\right\rangle=0$ whenever $\mathbb{P}(\{n=m\})=0$, and

- $\left\|x_{n}\right\|=1$ for all $n$.

An orthonormal sequence $\left(x_{n}\right)_{n \in L^{0}(\mathbb{N})}$ is generating if for every $x \in H$ there exists a sequence $\left(r_{n}\right)_{n \in L^{0}(\mathbb{N})}$ in $L^{0}(\mathcal{G}, \mathbb{R})$ such that $x=\sum_{n} r_{n} x_{n}{ }^{15}$. A generating orthonormal sequence is called an orthonormal basis.

The transfer principle applied to Avigad and Simic [1, Theorem 10.9] yields the following new result.

\footnotetext{
${ }^{14}$ See [1, Definition 9.3] for a definition in second-order arithmetic.

${ }^{15}$ Notice that this sum does not have a well-defined value in a standard setting a priori as it is uncountable, as a limit in $\mathcal{S}$ it is though meaningful, and this value can then be interpreted in a standard setting.
} 
Theorem 4.30 $L^{2}(\mathcal{F} \mid \mathcal{G})$ has an orthonormal basis in $\mathcal{S}$.

Remark 4.31 The significance of Theorem 4.30 lies in the fact that generally an $L^{0}(\mathbb{R})$-module with the countable concatenation property has an algebraic basis if and only if it is finitely ranked, see Jamneshan and Zapata [38, Proposition 3.5]. This means that an infinite dimensional space such as $L^{2}(\mathcal{F} \mid \mathcal{G})$ does not have any module linear basis in a standard sense in general ${ }^{16}$. The difference in $\mathcal{S}$ is that one allows the family which forms the basis to be a conditional family, cf [38, Section 3].

We have the following projection theorem thanks to the transfer principle and [1, Theorem 12.5].

Theorem 4.32 Let $W$ be a sequentially closed sub-module of $L^{2}(\mathcal{F} \mid \mathcal{G})$. Then every point $x \in L^{2}(\mathcal{F} \mid \mathcal{G})$ has a smallest distance $d(x, W):=\inf _{y \in W} d(x, y)$.

A consequence of the projection theorem is the orthogonal decomposition theorem:

Theorem 4.33 Let $W$ be a sequentially closed sub-module of $L^{2}(\mathcal{F} \mid \mathcal{G})$. Then there exists a sequentially closed sub-module $W^{\perp}$ such that each element $x \in L^{2}(\mathcal{F} \mid \mathcal{G})$ has a unique decomposition $x=z+y$ where $z \in W$ and $y \in W^{\perp}$.

We have also a Riesz representation theorem due to [1, Theorem 13.4] which interpreted in a standard setting reads as follows.

Theorem 4.34 Let $f: L^{2}(\mathcal{F} \mid \mathcal{G}) \rightarrow L^{0}(\mathbb{R})$ be an $L^{0}(\mathbb{R})$-linear and sequentially continuous function. Then there exists $y \in L^{2}(\mathcal{F} \mid \mathcal{G})$ such that $f(x)=\mathbb{E}[x y \mid \mathcal{G}]$.

We obtain the following extension of von Neumann's mean ergodic theorem, see [1] for the result in $\mathrm{ACA}_{0}$.

Theorem 4.35 Let $T: L^{2}(\mathcal{F} \mid \mathcal{G}) \rightarrow L^{2}(\mathcal{F} \mid \mathcal{G})$ be $L^{0}(\mathbb{R})$-linear such that $\|T x\| \leqslant\|x\|$ for all $x \in L^{2}(\mathcal{F} \mid \mathcal{G})$. Then $1 / n\left(x+T x+\ldots+T^{n-1} x\right)$ converges in the vector norm of $L^{2}(\mathcal{F} \mid \mathcal{G})$ almost everywhere to the projection of $x$ to the sequentially closed sub-module of all $T$-invariant vectors.

Remark 4.36 The conditional Hilbert space $L^{2}(\mathcal{F} \mid \mathcal{G})$ was introduced in Hansen and Richard [31] for purposes of financial modeling. Some applications in stochastic analysis of an orthogonal decomposition result are described in Cerreia-Vioglio et al [7]. A Riesz representation theorem, a projection theorem and an orthogonal decomposition theorem in complete random inner product spaces are proved in Guo [28, Section 4]. A mean ergodic theorem for complete random inner product spaces is established in Guo and Zhang [30].

\footnotetext{
${ }^{16}$ For instance, if $(\Omega, \mathcal{F}, \mathbb{P})$ is purely non-atomic.
} 


\subsection{Fixed point theorems}

We close this section with fixed point theorems in the 'conditional model' $\mathcal{S}$. One could think of applications in equilibrium theory and random differential equations.

A conditional version of the Brouwer fixed point theorem was proved in Drapeau et al [18]. The precise statement in the standard model is the following.

Theorem 4.38 Let $x_{1}, \ldots, x_{k}$ be finitely many points in $L^{0}(\mathbb{R})^{n}, n \in \mathbb{N}$. Let

$$
S=\left\{\sum_{i=1}^{k} \lambda_{i} x_{i}: 0 \leqslant \lambda_{i} \leqslant 1, i=1, \ldots, k\right\}
$$

Let $f: S \rightarrow S$ be a stable sequentially continuous function. Then there is $x \in S$ such that $f(x)=x$.

The proof in [18] is based on an adaptation of Sperner's lemma to a conditional setting. One obtains Sperner's lemma and the Brouwer fixed point theorem in the following slightly more general form as a consequence of the transfer principle applied to Simpson [47, Theorem IV.7.6].

Theorem 4.39 Let $k, n \in L^{0}(\mathbb{N})$ and $\left(x_{i}\right)_{i \leqslant k}$ be a conditionally finite sequence of points in $L^{0}(\mathbb{R})^{n}$. Without loss of generality suppose that $k=\sum_{m} k_{m} \mid A_{m}$ and $n=\sum_{m} n_{m} \mid A_{m}$, and let $\left(x_{i}^{m}\right)_{i \leqslant k_{m}}$ be finitely many elements in $L^{0}(\mathbb{R})^{n_{m}}$ which comprise the conditional sequence $\left(x_{i}\right)_{i \leqslant k}$ on $A_{m}$ for each $m$. Build the conditional simplex

$$
S:=\sum_{m}\left\{\sum_{i=1}^{k_{m}} \lambda_{i} x_{i}^{m}: 0 \leqslant \lambda_{i} \leqslant 1, i=1, \ldots, k_{m}\right\} \mid A_{m}
$$

Then every stable sequentially continuous function $f: S \rightarrow S$ has a fixed point.

As a novel result, we deduce a conditional version of the Markov-Kakutani fixed point theorem by applying the transfer principle to [47, Lemma IV.9.1].

Theorem 4.40 Let

$$
[-1,1]^{L^{0}(\mathbb{N})}:=\left\{\left(x_{k}\right)_{k \in L^{0}(\mathbb{N})}: x_{k} \in L^{0}(\mathbb{R}),-1 \leqslant x_{k} \leqslant 1 \text { for all } k\right\}
$$

endowed with the conditional metric ${ }^{17}$

$$
d\left(\left(x_{k}\right),\left(y_{k}\right)\right):=\sum_{k} 2^{-k} \frac{\left|x_{k}-y_{k}\right|}{\left|x_{k}-y_{k}\right|+1}
$$

\footnotetext{
${ }^{17}$ Recall that the sum is uncountable in a standard world, but makes sense in a 'conditional model' as the limit of conditionally finite sums.
} 
Let $S$ be a stable, sequentially closed and $L^{0}(\mathbb{R})$-convex ${ }^{18}$ subset of $[-1,1]^{L^{0}(\mathbb{N})}$. Let $\left(f_{k}\right)$ be a conditional sequence ${ }^{19}$ of $L^{0}(\mathbb{R})$-affine and sequentially continuous functions $f_{k}: S \rightarrow S$ such that $f_{k} \circ f_{m}=f_{m} \circ f_{k}$ for all $m, k \in L^{0}(\mathbb{N})$. Then there exists $x \in S$ such that $f_{k}(x)=x$ for all $k$.

\section{Further connections and outlook}

We have left out measure theory. For the development of measure theory in second-order arithmetic, we refer to Simic [46], Simpson [47] and Yu [52]. In conditional set theory, basic results in measure theory are established in Jamneshan et al [36]. As measure theory in second-order arithmetic is based on Daniell's functional approach to integration, the conditional version of the Daniell-Stone theorem and Riesz representation theorem in [36, Section 5] can be connected to respective theorems in $\mathcal{S}$. In [36, Section 4], a connection between kernels in a standard model and measures in conditional set theory are proved. This connection fully carries over to the model $\mathcal{S}$, for Borel probability measures on $H$ where $H$ is a conditionally compact metric space. Perspectively, understanding the standard meaning of the existence of a Haar measure [47, Section X.1], and of the maximal ergodic and pointwise ergodic theorems [46, Chapter 5] in $\mathcal{S}$ may be of interest.

For some mathematical theorems relevant for applications in mathematical economics, stronger second-order axioms than $\mathrm{ACA}_{0}$ are required. For example, weak*-closures play an important role in a recent duality result in vector optimization in Grad and Jamneshan [26] which is useful for applications such as minimization of conditional risk measures. Now, by [47, Theorem X.2.9], $\Pi_{1}^{1}-\mathrm{CA}_{0}$ is required to prove that weak ${ }^{*}$ closures in the normal dual of a separable Banach space even exist. Moreover, it has been argued eg by Kohlenbach in [41] that the second-order setting, comprehensive as it is, is not sufficient for important areas like functional analysis or topology, and should therefore be extended to higher-order frameworks that also allow for quantification over sets of real numbers, sets of sets of real numbers, sets thereof, et cetera.

It would thus be worthwhile (1) to consider the validity of stronger comprehension axioms like $\Pi_{1}^{1}-\mathrm{CA}_{0}$ (see [47]) or, more generally, $\Pi_{1}^{n}-\mathrm{CA}_{0}$ in our conditional model, and (2) to come up with conditional interpretations of higher-order systems and a corresponding transfer principle. The question whether $\Pi_{1}^{1}-\mathrm{CA}_{0}$ holds in our model is

\footnotetext{
${ }^{18}$ This means $\lambda x+(1-\lambda) y \in S$ whenever $x, y \in S$ and $\lambda \in L^{0}(\mathbb{R})$ with $0 \leqslant \lambda \leqslant 1$.

${ }^{19}$ This means for functions that $\left(\sum_{n} f_{k_{n}} \mid A_{n}\right)(x)=\sum_{n} f_{k_{n}}(x) \mid A_{n}$ for all $x$, every sequence $\left(k_{n}\right)$ in $L^{0}(\mathbb{N})$ and measurable partition $\left(A_{n}\right)$.
} 
not immediately answered by the method used above for $\mathrm{ACA}_{0}$. For this purpose, it would be necessary to consider projections of sets of real numbers, which would require working in a conditional power set of the conditional real numbers.

Nevertheless, we are optimistic that such extensions can be constructed. Indeed, following the general construction of a conditional power set in [15], more precisely its variant defined in [36], the conditional power set of the conditional power set of the conditional natural numbers can be formed, which indicates the necessary construction needed to prove the validity of stronger comprehension schemes and a transfer principle for higher-order systems. We plan to develop this in detail in future work.

\section{References}

[1] J Avigad, K Simic, Fundamental notions of analysis in subsystems of second-order arithmetic, Ann. Pure Appl. Logic 139 (2006) 138-184; https://doi.org/10.1016/j.apal. 2005.03.004

[2] A Aviles, J M Zapata, Boolean-valued Models as a Foundation for Locally $L^{0}$-Convex Analysis and Conditional Set Theory, IFCoLog Journal of Logic and its Applications 5 (2018) 389-420

[3] J Backhoff, U Horst, Conditional analysis and a Principal-Agent problem, SIAM J. Financial Math. 7 (2016) 477-507; https://doi.org/10.1137/14100066X

[4] J L Bell, Set Theory: Boolean-Valued Models and Independence Proofs, Oxford Logic Guides, Clarendon Press (2005); https://doi.org/10.2307/2273926

[5] T Bielecki, I Cialenco, S Drapeau, M Karliczek, Dynamic Assessement Indices, Stochastics 88 (2016) 1-44; https://doi.org/10.1080/17442508.2015.1026346

[6] S Cerreia-Vioglio, M Kupper, F Maccheroni, M Marinacci, N Vogelpoth, Conditional $L_{p}$-spaces and the duality of modules over $f$-algebras, J. Math. Anal. Appl. 444 (2016) 1045-1070; https://doi.org/10.1016/j.jmaa.2016.06.018

[7] S Cerreia-Vioglio, F Maccheroni, M Marinacci, Orthogonal Decompositions in Hilbert A-Modules, Preprint (2017)

[8] P Cheridito, U Horst, M Kupper, T Pirvu, Equilibrium pricing in incomplete markets under translation invariant preferences, Math. Oper. Res. 41 (2016) 174-195; https://doi.org/10.1287/moor.2015.0721

[9] P Cheridito, Y Hu, Optimal consumption and investment in incomplete markets with general constraints, Stoch. Dyn. 11 (2011) 283-299; https://doi.org/10.1142/S0219493 711003280

[10] P Cheridito, M Kupper, N Vogelpoth, Conditional analysis on $\mathbb{R}^{d}$, Set Optimization and Applications, Proceedings in Mathematics \& Statistics 151 (2015) 179-211; https://doi.org/10.1007/978-3-662-48670-2 
[11] P Cheridito, M Stadje, BS $\triangle$ Es and BSDEs with non-Lipschitz drivers: comparison, convergence and robustness, Bernoulli 19 (2013) 1047-1085; https://doi.org/10.3150/12BEJ445

[12] K Detlefsen, G Scandolo, Conditional and Dynamic Convex Risk Measure, Finance Stoch. 9 (2005) 539-561; https://doi.org/10.1007/s00780-005-0159-6

[13] J Diestel, J J Uhl, Vector Measures, Mathematical surveys and monographs, American Mathematical Society (1977); https://doi.org/10.1090/surv/015

[14] S Drapeau, A Jamneshan, Conditional preferences and their numerical representations, J. Math. Econom. 63 (2016) 106-118; https://doi.org/10.1016/j.jmateco.2015.12.004

[15] S Drapeau, A Jamneshan, M Karliczek, M Kupper, The algebra of conditional sets and the concepts of conditional topology and compactness, J. Math. Anal. Appl. 437 (2016) 561-589; https://doi.org/10.1016/j.jmaa.2015.11.057

[16] S Drapeau, A Jamneshan, M Kupper, Vector duality via conditional extension of dual pairs (2016); arXiv: 1608.08709

[17] S Drapeau, A Jamneshan, M Kupper, A Fenchel-Moreau theorem for $\bar{L}^{0}$-valued functions, J. Convex Anal. (to appear)

[18] S Drapeau, M Karliczek, M Kupper, M Streckfuss, Brouwer fixed point theorem in $\left(L^{0}\right)^{d}$, J. Fixed Point Theory Appl. 301 (2013); https://doi.org/10.1186/1687-1812-2013301

[19] D Filipović, M Kupper, N Vogelpoth, Separation and duality in locally $L^{0}$-convex modules, J. Funct. Anal. 256 (2009) 3996-4029; https://doi.org/10.1016/j.jfa.2008.11.015

[20] D Filipović, M Kupper, N Vogelpoth, Approaches to conditional risk, SIAM J. Financial Math. 3(1) (2012) 402-432; https://doi.org/10.1137/090773076

[21] H Föllmer, A Schied, Stochastic Finance. An Introduction in Discrete Time, 3 edition, de Gruyter Studies in Mathematics, Walter de Gruyter, Berlin, New York (2011); https://doi.org/10.1515/9783110218053

[22] D Fremlin, Topological Riesz Spaces and Measure Theory, Cambridge University Press (1974); https://doi.org/10.1017/CBO9780511897207

[23] M Frittelli, M Maggis, Conditional Certainty Equivalent, Int. J. Theor. Appl. Finance 14 (2011) 41-59; https://doi.org/10.1142/S0219024911006255

[24] M Frittelli, M Maggis, Complete duality for quasiconvex dynamic risk measures on modules of the $L^{p}$-type, Stat. Risk Model. 31 (2014) 103-128; https://doi.org/10.1515/strm2013-1163

[25] S R Givant, P R Halmos, Introduction to Boolean Algebras, Undergraduate texts in mathematics, Springer (2009); https://doi.org/10.1007/978-0-387-68436-9

[26] S-M Grad, A Jamneshan, A perturbational duality approach in vector optimization (2018); arXiv:1807.02666 
[27] T Guo, Relations between some basic results derived from two kinds of topologies for a random locally convex module, J. Funct. Anal. 258 (2010) 3024-3047; https://doi.org/10.1016/j.jfa.2010.02.002

[28] T Guo, On Some Basic Theorems of Continuous Module Homomorphisms between Random Normed Modules, J. Funct. Spaces (2013) 1-13; https://doi.org/10.1155/2013 1989102

[29] T Guo, H Xiao, X Chen, A basic strict separation theorem in random locally convex modules, Nonlinear Anal. Real World Appl. 71 (2009) 3794-3804; https : //doi .org/ 10.1016/j.na.2009.02.038

[30] T Guo, X Zhang, Von Neumann's mean ergodic theorem on complete random inner product modules, Front. Math. China 6 (2011) 965-985; https://doi.org/10.1007/s11464011-0139-4

[31] L P Hansen, S F Richard, The Role of Conditioning Information in Deducing Testable Restrictions Implied by Dynamic Asset Pricing Models, Econometrica 55 (1987) 587613; https://doi.org/10.2307/1913601

[32] R Haydon, M Levy, Y Raynaud, Randomly Normed Spaces, Hermann (1991)

[33] T Hytönen, J van Neerven, M Veraar, L Weis, Analysis in Banach Spaces: Volume I: Martingales and Littlewood-Paley Theory, Ergebnisse der Mathematik und ihrer Grenzgebiete. 3. Folge / A Series of Modern Surveys in Mathematics, Springer International Publishing (2016); https://doi.org/10.1007/978-3-319-48520-1

[34] A Jamneshan, A theory of conditional sets, $\mathrm{PhD}$ thesis, Humboldt-Universität zu Berlin, Mathematisch-Naturwissenschaftliche Fakultät II (2014)

[35] A Jamneshan, Sheaves and conditional sets (2014); arXiv:1403.5405

[36] A Jamneshan, M Kupper, M Streckfuß, Measures and integrals in conditional set theory, Set-Valued Var. Anal. (to appear); https://doi.org/10.1007/s11228-018-0478-3

[37] A Jamneshan, M Kupper, J M Zapata, Parameter-dependent stochastic optimal control in finite discrete time (2017); arXiv: 1705.02374

[38] A Jamneshan, JM Zapata, On compactness in $L^{0}$-modules (2017); arXiv: 1711.09785

[39] Y Kabanov, C Stricker, A teacher's note on no-arbitrage criteria, Séminaire de Probabilités 35 (2001) 149-152; https://doi.org/10.1007/b76885

[40] A Kechris, Classical Descriptive Set Theory, Graduate Texts in Mathematics, SpringerVerlag (1995); https://doi.org/10.1007/978-1-4612-4190-4

[41] U Kohlenbach, Higher order reverse mathematics, In: Stephen G. Simpson (ed.) Reverse Mathematics 2001. Lecture Notes in Logic vol. 21, ASL, A K Peters (2005) 281-295; https://doi.org/10.1017/9781316755846.018

[42] A G Kusraev, S S Kutateladze, Boolean Valued Analysis, Mathematics and Its Applications, Springer Netherlands (2012); https://doi.org/10.13140/RG.2.1.2164.6486 
[43] S S Kutateladze, Nonstandard Analysis and Vector Lattices, Mathematics and Its Applications, Springer Netherlands (2012); https://doi.org/10.1007/978-94-011-4305-9

[44] I Molchanov, Theory of Random Sets, Probability and Its Applications, Springer London (2005); https://doi.org/10.1007/1-84628-150-4

[45] R T Rockafellar, R J-B Wets, Variational Analysis, Springer, Berlin, New York (1998); https://doi.org/10.1007/978-3-642-02431-3

[46] K Simic, Aspects of Ergodic Theory in Subsystems of Second-order Arithmetic, $\mathrm{PhD}$ thesis, Carnegie Mellon University (2004)

[47] S G Simpson, Subsystems of Second Order Arithmetic, Perspectives in Mathematical Logic, Springer-Verlag Berlin Heidelberg (1999); https://doi.org/10.1017/CBO9 780511581007

[48] A Skorohod, Random Linear Operators, Mathematics and its Applications, Springer Netherlands (1983)

[49] J Strand, Random ordinary differential equations, J. Differential Equations 7 (1970) 538-553; https://doi.org/10.1016/0022-0396(70)90100-2

[50] G Takeuti, Proof Theory, Dover books on mathematics, Dover Publications, Incorporated (2013)

[51] N Vogelpoth, $L^{0}$-convex Analysis and Conditional Risk Measures, $\mathrm{PhD}$ thesis, University of Vienna (2009)

[52] X Yu, Riesz representation theorem, Borel measures and subsystems of second-order arithmetic, Ann. Pure Appl. Logic 59 (1993) 65-78; https://doi.org/10.1016/01680072(93)90232-3

Department of Mathematics and Statistics, University of Konstanz

78457 Konstanz, DE

merlin.carl@uni-konstanz.de, asgar.jam@gmail.com

Received: 4 April 2018 Revised: 4 October 2018 